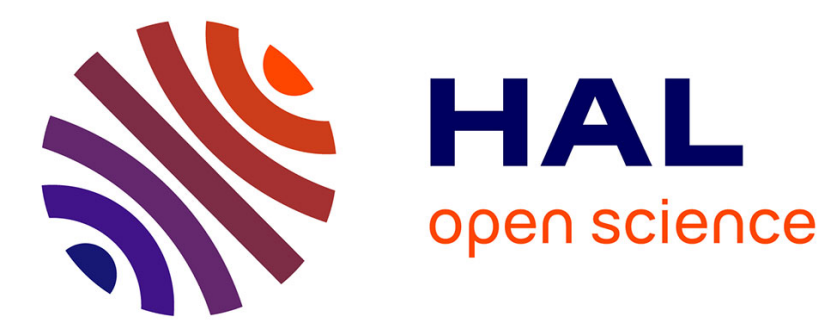

\title{
Determination of heliostat canting errors via deterministic optimization
}

Alberto Sánchez-González, Cyril Caliot, Alain Ferrière, Domingo Santana

\section{To cite this version:}

Alberto Sánchez-González, Cyril Caliot, Alain Ferrière, Domingo Santana. Determination of heliostat canting errors via deterministic optimization. Solar Energy, 2017, 150, pp.136 - 146. 10.1016/j.solener.2017.04.039 . hal-03368189

\section{HAL Id: hal-03368189 \\ https://hal.science/hal-03368189}

Submitted on 7 Oct 2021

HAL is a multi-disciplinary open access archive for the deposit and dissemination of scientific research documents, whether they are published or not. The documents may come from teaching and research institutions in France or abroad, or from public or private research centers.
L'archive ouverte pluridisciplinaire $\mathbf{H A L}$, est destinée au dépôt et à la diffusion de documents scientifiques de niveau recherche, publiés ou non, émanant des établissements d'enseignement et de recherche français ou étrangers, des laboratoires publics ou privés. 


\title{
Determination of heliostat canting errors via deterministic optimization
}

\author{
Alberto Sánchez-González ${ }^{\mathrm{a}, *}$, Cyril Caliot $^{\mathrm{b}}$, Alain Ferrière ${ }^{\mathrm{b}}$, Domingo Santana ${ }^{\mathrm{a}}$ \\ ${ }^{a}$ Universidad Carlos III de Madrid, Department of Thermal and Fluid Engineering, Av. Universidad, 30, 28911 Leganés, Madrid, Spain \\ ${ }^{\mathrm{b}}$ Processes, Materials and Solar Energy Laboratory, PROMES-CNRS, 7 rue du Four Solaire, 66120 Font-Romeu-Odeillo-Via, France
}

\section{A R T I C L E I N F O}

\section{Article history:}

Received 10 November 2016

Received in revised form 16 March 2017

Accepted 17 April 2017

Available online 24 April 2017

\section{Keywords:}

Solar power tower

Heliostat optical quality

Flux distribution

DIRECT algorithm

\begin{abstract}
A B S T R A C T
This paper presents a novel methodology to find out canting errors in the facets, i.e. mirror modules, of heliostats. An optimization procedure is established to fit simulated heliostat flux distributions to those captured on a white target. On the basis of a convolution-projection optical model, a deterministic algorithm - named DIRECT - has been successfully implemented, reaching correlation coefficients up to 95.8\%. In this instance, the procedure has been applied to a THEMIS heliostat presenting canting errors of its faceted modules. From the optimization results, the heliostat modules were accordingly readjusted. And the heliostat optical quality has been significantly increased, validating the proposed methodology. (c) 2017 Elsevier Ltd. All rights reserved.
\end{abstract}

\section{Introduction}

Beam quality of heliostats depends on correct alignment. Heliostat alignment involves two operations: mirror focusing and heliostat canting. Mirror focusing consists in slightly bending the mirror surface into a concave shape, so that the size of the reflected sun image is minimized (Chong, 2014). Heliostat canting consists in tilting the mirror modules to aim at the same point. Proper heliostat alignment results in maximizing the annual power intercepted by the receiver (Jones, 1996b).

Heliostat canting techniques, on which the present study is focused, are classified into three categories (Ren et al., 2014): onsun, mechanical, and optical alignment. In the first method, mirror modules are individually - and qualitatively - canted while the sun is impinging on the heliostat and the rest of the modules are covered. Mechanical alignment makes use of gauge blocks or inclinometers to adjust the orientation of the modules while the heliostat is in horizontal position; this method is very time consuming, just like on-sun alignment.

Six optical alignment techniques can be identified: laser method, camera look-back, photogrammetry, deflectometry, TOPHAT and H-FACET. There are two types of laser beam projection methods (Yellowhair and Ho, 2010): scanning prism laser projection and parallel laser beam projection. Camera look-back method was developed and successfully tested by SNL (Jones et al., 1994). Photogrammetry and deflectometry techniques utilize

\footnotetext{
* Corresponding author.

E-mail addresses: asgonzal@ing.uc3m.es (A. Sánchez-González), cyril.caliot@ promes.cnrs.fr (C. Caliot).
}

camera images to determine the orientation of heliostat facets. Theoretical overlay photographic heliostat alignment technique, TOPHAT (SNL, 2013), and heliostat focusing and canting enhancement technique, H-FACET (Sproul et al., 2011), are tools feed by camera images, both of which have been recently developed by SNL.

In this paper it is proposed a novel methodology to find out canting errors in focused heliostats to correct them. From heliostat experimental images taken in THEMIS solar power tower plant (research and development center operated by CNRS at Targasonne, France), an optimization procedure has been developed to minimize the difference between experimental flux distribution and simulations, where canting errors in the modules are the unknowns. The experimental set up and heliostat characteristics are described in the next section. Afterwards, the proposed procedure is described, and results for a heliostat with low optical quality are presented. From the results, the selected heliostat has been in situ readjusted to validate the proposed methodology.

\section{Problem description}

Misalignment of mirror facets leads to heliostats with poor optical quality. On a lambertian target near the receiver, flux distributions from misaligned heliostats result in images with multiple spots. To develop and validate a method to correct misaligned heliostat faceted modules, we use an heliostat from the THEMIS solar facility presenting canting errors. This section describes the characteristics of the heliostat and the experimental campaign carried out at THEMIS. 


\begin{tabular}{|c|c|c|c|}
\hline \multicolumn{4}{|c|}{ Nomenclature } \\
\hline CCC & cross-correlation coefficient [-] & $\mathrm{h}$ & heliostat \\
\hline$F$ & flux density $\left[\mathrm{W} / \mathrm{m}^{2}\right]$ & $\mathrm{m}$ & module \\
\hline$f$ & focal distance $[\mathrm{m}]$ & $\max$ & maximum \\
\hline FN & normalized flux density $[-]$ & $\bmod$ & model \\
\hline IL & intensity level [-] & $\operatorname{slp}$ & slope \\
\hline$N$ & number [\#] & sun & sunshape \\
\hline n & heliostat normal vector & & \\
\hline RMSD & root mean square deviation [-] & \multicolumn{2}{|l|}{ Acronyms } \\
\hline $\mathbf{s}$ & sun vector & CCD & Charge-Coupled Device \\
\hline$S D$ & standard deviation & CEST & Central European Summer Time \\
\hline t & target vector & CNRS & Centre National de la Recherche Scientifique \\
\hline TP & position of the target point & CRS2 & Controlled Random Search, version 2 \\
\hline WC & position of the weighted centroid & DIRECT & DIviding RECTangles \\
\hline$X, Y, Z$ & Cartesian coordinate axes & H-FACET & $\begin{array}{l}\text { Heliostat Focusing And Canting Enhancement } \\
\text { Technique }\end{array}$ \\
\hline \multicolumn{2}{|c|}{ Greek symbols } & MCRT & Monte Carlo Ray Tracing \\
\hline$\delta$ & angular canting deviation [mrad] & PROMES & PROcédés, Matériaux et Énergie Solaire \\
\hline \multirow[t]{2}{*}{$\sigma$} & Gaussian error [mrad] & SNL & Sandia National Laboratories \\
\hline & & TOPCAT & Theoretical Overlay Photographic Heliostat Alignment \\
\hline \multicolumn{2}{|c|}{ Subscripts } & & Technique \\
\hline elts & elements & UTC & Coordinated Universal Time \\
\hline exp & experimental & & \\
\hline
\end{tabular}

\subsection{Canting errors in CETHEL heliostats}

Heliostats in THEMIS field, whose model is named CETHEL, consist of 9 rectangular modules of mirrors. Eight modules are distributed in two wings at either side of the heliostat pole, and a ninth smaller module is in between and above the pole. Fig. 1 shows a photograph of one of the heliostats in the field (a) and a drawing with the dimensions of the heliostat and the modules (b). Total reflective surface is $54 \mathrm{~m}^{2}$.

The module consists of three vertical strips of parabolic mirror; two strips in the complimentary module. Each mirror strip is mechanically tighten to the module frame so that curvature and orientation are forced. As a result, each module acts almost like a single spherical mirror with focal distance, $f_{m}$. This way, heliostat focusing is achieved. In this study, CETHEL heliostats are assumed to be properly focused.
Surface slope error $\left(\sigma_{s l p}\right)$, defined as the root mean square error of the true to the ideal spherical mirror shape, is not known. For CETHEL heliostats mirror slope error is expected to be around 1 mrad.

On the other hand, each module is supported on the rear heliostat structure in three points. Through adjustment of these three screw-nut assemblies, each module can be slightly tilted along its two axes; only one in the case of the complimentary module. This process is known as heliostat canting.

A heliostat is properly canted when the normal vectors of all the modules intersect in the same point. This point is the center of a sphere with radius $2 \cdot f_{h}$, being $f_{h}$ the focal length of the heliostat. Fig. 2 represents the geometry of CETHEL heliostat with modules canted towards the same point. This is called on-axis alignment, because it is optimized for the case of heliostat center, target and sun falling in the same line; otherwise, the term off-axis alignment is utilized (Jones, 1996a).

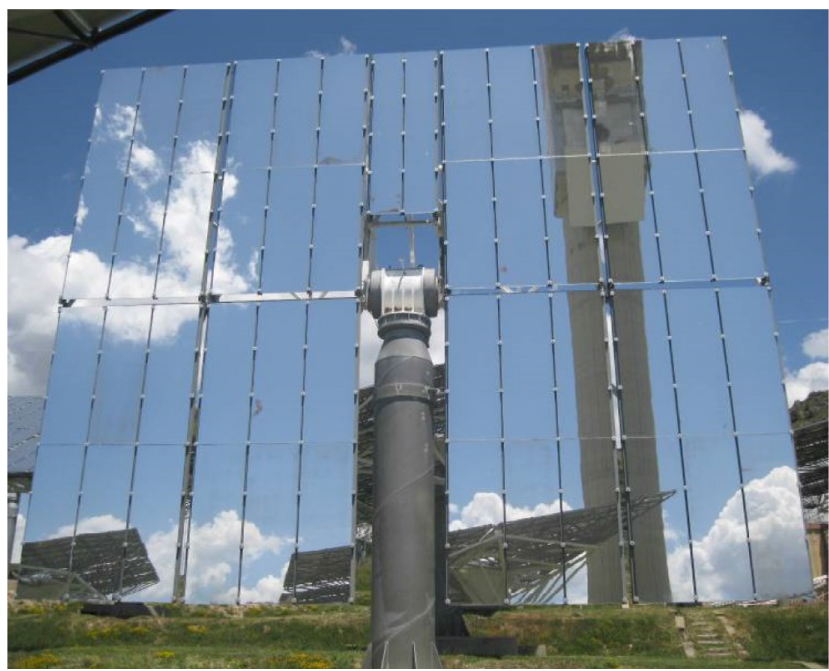

(a) Front view photograph.

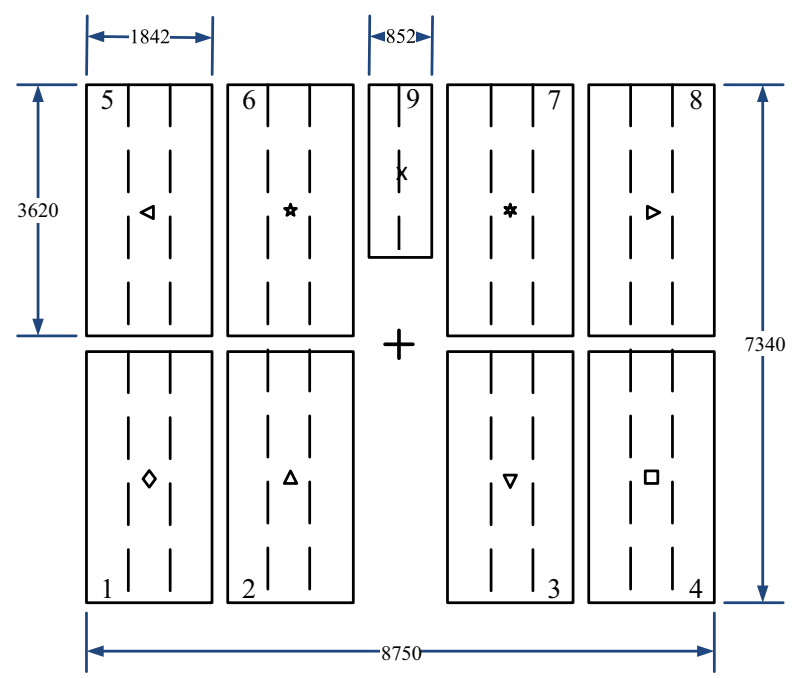

(b) Geometry. Dimensions in mm.

Fig. 1. CETHEL heliostat. 


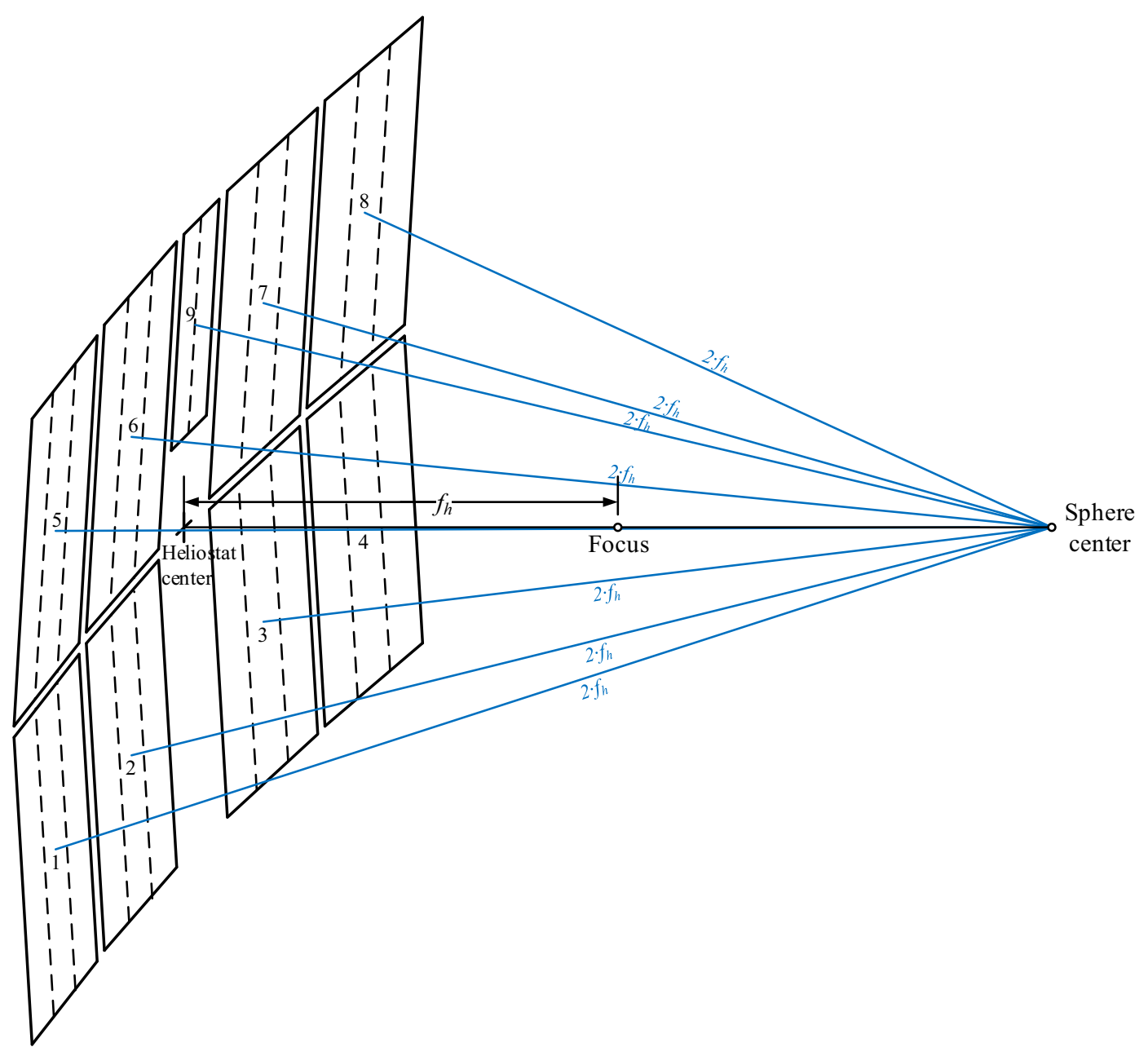

Fig. 2. On-axis aligned CETHEL heliostat.

CETHEL heliostats were initially canted during THEMIS plant commissioning in early 80 's. A bubble leveled tool was used to cant each module while the heliostat is in horizontal position. More than 30 years later, some of the heliostats may have lost optical quality, according to flux images on the target plane; as will be shown later.

The source of current lack of adjustment is not known with certainty, but some hypothesis can be made. An initial guess is the progressive loosen of screw nuts that keeps the module in a fixed position. Another hypothesis is the presence of permanent deformation in the supporting structure due to the impact of hail or even provoked by extreme wind gusts. From this point of view, THEMIS heliostats present a deterministic canting error that can be attributed to aging and hail.

Elastic deformation of heliostat structure because of gravity sag (Yuan et al., 2014) is presumed to have small influence on the optical quality of CETHEL heliostats, because of its structural stiffness. By means of photogrammetry it has been previously demonstrated the slight influence of actual shape on flux distribution (Mutuberria et al., 2012). Within the accuracy limits of the proposed method, it is reasonable to neglect the influence of the deformation due to gravity sag. Secondly, the experimental campaign was carried out along a windless day, to avoid such source of deformation.

Angular canting deviations, $\delta$, are referred to the ideal orientation of the module occurring when it is properly canted. The reference coordinate system of the module with origin in its center is depicted in Fig. 3; $X$ is parallel to the module short side, $Y$ is parallel to the long side, and $Z$ is perpendicular to the mirror and oriented towards the center of the sphere with radius $2 \cdot f_{h}$.

On the basis of the coordinate system, two angles are defined to describe the error of canting in each module: $\delta_{x}$ rotation angle about $X$ axis, and $\delta_{y}$ rotation angle about $Y$ axis. In module 9 , canting is only allowed about the horizontal axis, $\delta_{x}$. Therefore, a total of 17 angular canting errors can be defined.

In this paper, it is exposed a methodology to find out those 17 canting errors for CETHEL heliostats. Slope error, assumed uniform for all the heliostat modules, is also unknown. In total, there are 18 unknowns in the problem. The proposed solution method is based on an optimization procedure which makes use of experimental images as the basic input in the model.

\subsection{Experimental campaign}

THEMIS plant is located in Targasonne, France, at $42^{\circ} 30^{\prime}$ north latitude and $1^{\circ} 58^{\prime}$ east longitude. THEMIS solar power tower plant was under commercial operation in the period 1983-1986, and currently is a solar research platform belonging to CNRS, PROMES laboratory.

THEMIS solar field consists of 107 CETHEL heliostats placed on a south oriented hillside. The heliostat field follows a radial staggered north field arrangement. On the top of a $100 \mathrm{~m}$ high tower, two open areas allow the testing of flat, cavity and volumetric receivers. 


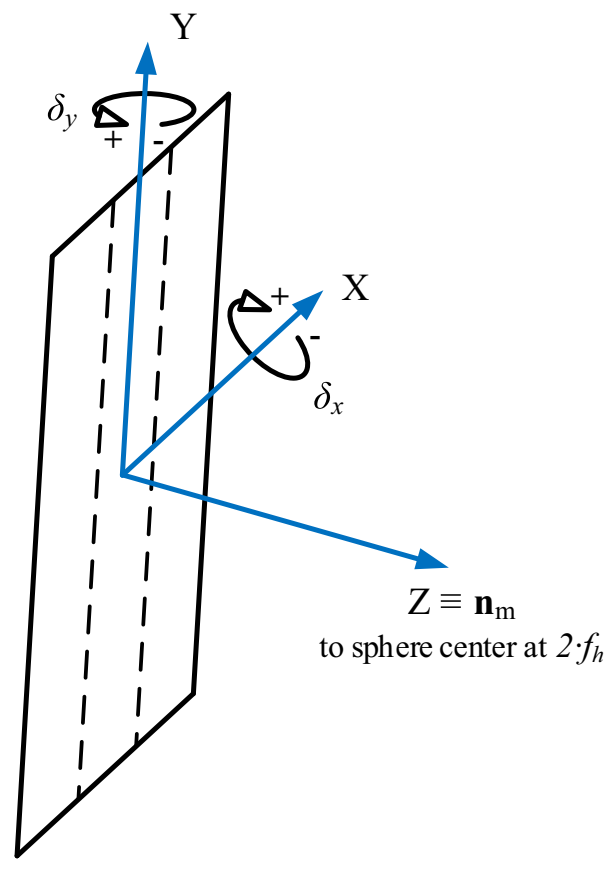

Fig. 3. Module system of coordinates for angular canting errors.

To analyze the optical quality of heliostats, they are aimed to the white target placed under the receiver apertures at $68.3 \mathrm{~m}$ from the base of the tower. The square lambertian target, $7.5 \mathrm{~m}$ side and tilted from the vertical $30^{\circ}$ towards the heliostat field, has the property of diffusively reflecting flux, regardless of the point of view.

On the ground, in the middle of the heliostat field, a camera with CCD sensor is utilized to take pictures of the flux distribution reflected by the white target. The CCD camera with adapted filters has $1392 \times 1040$ pixels of spatial resolution and each pixel has 14 bits or, equivalently, 16384 levels. Rather than $F$ flux density measurement in $\mathrm{W} / \mathrm{m}^{2}$, camera images provide intensity levels, IL. Dividing the level of intensity in each pixel by the maximum in an image, it is obtained an adimensional parameter equivalent to the normalized flux density, $F N$, defined as:

$F N=\frac{F}{F_{\max }} \equiv \frac{I L}{I L_{\max }}$

An experimental campaign was carried out along July 18, 2016. Several images from some heliostats were captured on the white target. Fig. 4 shows an aerial photo of THEMIS field taken during the experimental campaign.

Heliostat at position A06, labeled in Fig. 4, offered low optical quality in comparison with the remaining. Fig. 6 shows in dashed lines the experimental flux distributions from this heliostat at 11 instants of time. The multi-spot pattern is a typical feature of incorrectly canted heliostats. Consequently, heliostat A06 was selected to be readjusted according to the results from the proposed methodology.

A total amount of 28 images from heliostat A06 were recorded during 8 sunshine hours; on average, about one image every $20 \mathrm{~min}$. This means more images than the 17 degrees of freedom in the optimization problem, which reduces the uncertainty in the solution and improves its reliability.

Heliostat A06 is located at a distance of $89.76 \mathrm{~m}$ from the center of the white target; $X Y Z$ coordinates: $35.6270,-6.2753,82.1508 \mathrm{~m}$. As stated in heliostat A06 design specifications, the focal lengths of the heliostat, $f_{h}$, and the modules/mirrors, $f_{m}$, are respectively 103.6 and $100 \mathrm{~m}$. Because of the difference between focal lengths and slant range, some defocus - around $12 \mathrm{~m}$ - is expected on the white target.

\subsubsection{Image treatment}

Subsequently, raw images were processed to be handled by the computational model. Image treatment involved the operations of window selection, reconstruction of bad pixels and resolution adjustment. These operations were automated in a computer code.

The frame of the camera was larger than the $7.5 \times 7.5 \mathrm{~m}$ of the white target, thus images were initially cropped to match the target extension. Since captured flux spots were smaller than the size of the target, a centered window frame of $5 \times 5 \mathrm{~m}$ was finally taken. In this way, many pixels with zero intensity level are eliminated and computation time is saved later on with the model.

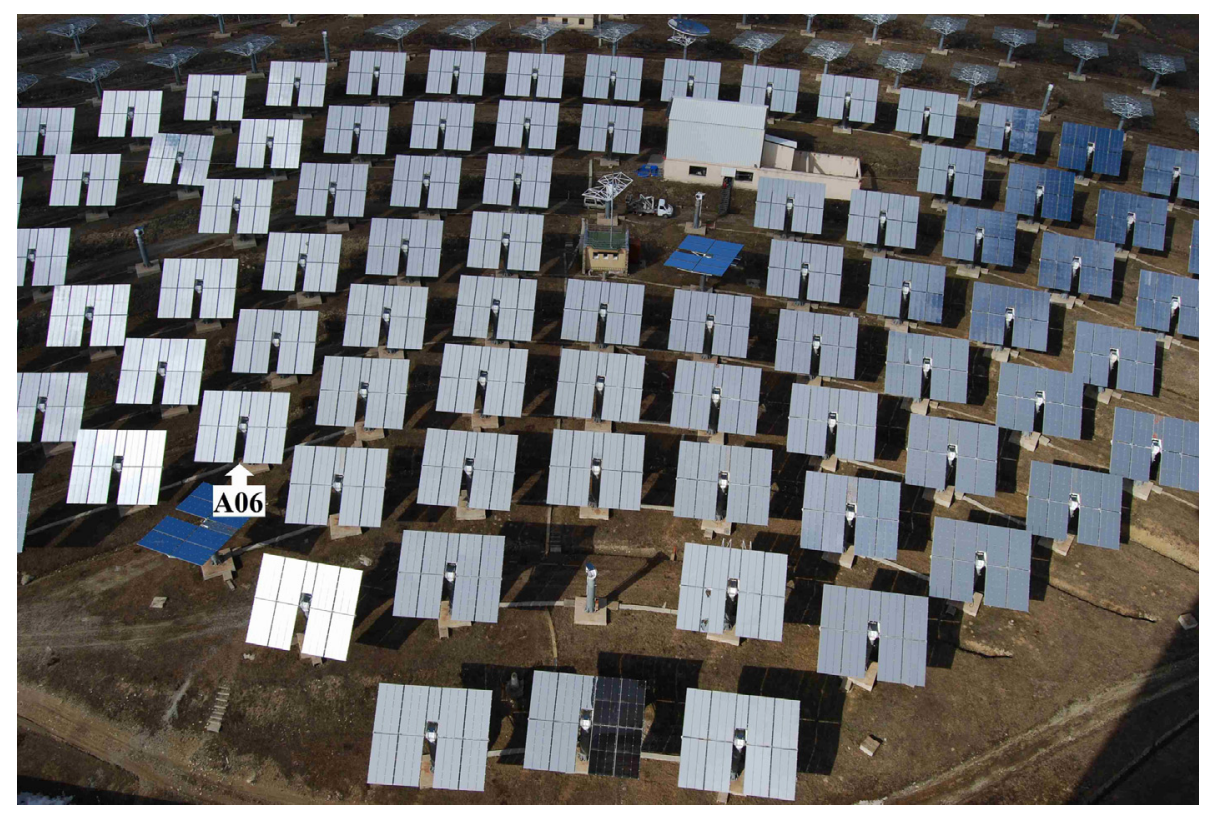

Fig. 4. Photograph of THEMIS heliostat field from the top of the tower. Heliostat A06 is labeled. 
From detailed image inspection, it was detected a tiny group of camera pixels giving erroneous values; usually smaller than expected. Once these pixels were identified, their values were removed and interpolation from surrounding pixels was performed.

Finally, resolution of the experimental image was forced to match that computed later in the model. Using the same mesh, the calculation of differences between experimental and simulated maps is faster and more efficient.

\section{Methodology}

The method developed to find out canting errors in heliostats is based on fitting the flux distributions from an accurate optical model to match the experimental images. The optical model incorporates the canting errors, that are the unknowns to be determined using an optimization procedure.

After introducing the optical model, this section describes the process to infer the heliostat target point at each instant of time. Later, the definition of the optimization method is presented: objective function, and selection of the optimization algorithm.

\subsection{Optical model}

Flux density distributions on the target are computed with the optical model previously validated by the authors (Sánchez-González and Santana, 2015). This model is based on the oblique projection, from image to target plane, of the flux distribution predicted by an analytic function derived from the convolution approach. In this instance, UNIZAR function by Collado et al. (1986) is utilized because of its accuracy with rectangular focused mirrors.

Instead of a single function evaluation per heliostat, the flux distribution by each rectangular module is computed separately and summed at the end. The angular canting errors in each module, $\delta_{x}$ and $\delta_{y}$, are taken into account in the calculation. For the 9 modules CETHEL heliostat, a total of 17 degrees of freedom are included in the model.

Besides steady geometric parameters like those presented in previous section, the model is fed with the sun position vector, $\mathbf{s}$. From the local clock time, UTC+2 (i.e. CEST), at which each image was captured, solar position has been gathered via the online calculator provided by the U.S. Naval Observatory (USNO, 2016).

For the sunshape, it has been adopted a Gaussian distribution with standard deviation, $\sigma_{\text {sun }}, 2.09 \mathrm{mrad}$, as used in other cone optics codes like HELIOS (Biggs and Vittitoe, 1979) and HFLCAL (Schmitz et al., 2006).

\subsection{Inference of the target point}

Canting angular errors, $\delta$, are referred to the ideal orientation of the modules taking place when they are on-axis aligned, as previously described in subSection 2.1. Ultimately, angular errors are referenced to the heliostat normal, that is a vector changing in time because of solar tracking.

Heliostat control algorithm in THEMIS works with encoder counts in both azimuth and elevation drives. However the reference biases of the encoders in the heliostat are ignored. Hence, an accurate measurement of instantaneous heliostat orientation (i.e. azimuth and elevation) is absent. To solve the lack of such datum, an inference procedure has been developed. Provided that the actual positions of the flux maps on the white target are known, the calculation of the target point is straightforward in the proposed procedure.

Given a solar position, defined by sun vector $\mathbf{s}$, the heliostat normal vector $(\mathbf{n})$ is found from the target vector $(\mathbf{t})$, and vice versa;
Eq. (2). The heliostat target point is in the intersection between vector $\mathbf{t}$ and the plane of the target. If the position of the heliostat target point could be deduced on the basis of the experimental image and the optical model, vectors $\mathbf{t}$ and $\mathbf{n}$ would be defined.

$\mathbf{n}=\frac{\mathbf{s}+\mathbf{t}}{|\mathbf{s}+\mathbf{t}|}$

The procedure to infer the position of the target point, TP, is based on matching the weighted centroids of the experimental image, $\mathbf{W} \mathbf{C}_{\text {exp }}$, and the simulated flux map, $\mathbf{W} \mathbf{C}_{\text {mod }}$. Taking $\mathbf{W} \mathbf{C}_{\text {exp }}$ as the initial target point to compute the flux map, an iterative procedure is established to force $\mathbf{W} \mathbf{C}_{\text {mod }}$ to coincide with $\mathbf{W} \mathbf{C}_{\text {exp }}$. In practice, just a single iteration is enough to reach an accurate estimation of the target point. This process is made at each instant of time, since the target point may be shifted from one image to the next.

Thus, the procedure to infer the position of the aim point on the target at each time instant can be defined in a simple 3-step process, as follows:

1. Calculate the centroid of the experimental image, $\mathbf{W C}_{\text {exp }}$.

2. With target point in $\mathbf{W} \mathbf{C}_{\text {exp }}$, simulate the flux distribution and calculate its centroid, $\mathbf{W C}_{\text {mod }}$.

3. Shift the target point to $\mathbf{W} \mathbf{C}_{\text {exp }}-\mathbf{W} \mathbf{C}_{\text {mod }}$.

The 3-step procedure to infer the heliostat aim point on the target plane is graphically represented in Fig. 5; where the experimental image was captured at 11:36 on July 18, 2016. Position vectors of the centroids and the target point are marked in the Figure. Taking advantage of vectorial notation, the position vector of the target point - step 3 - is therefore calculated with the following equation:

$\mathbf{T P}=2 \cdot \mathbf{W C}_{\exp }-\mathbf{W} \mathbf{C}_{\bmod }$

\subsection{Objective function}

When dealing with optimization problems, two major decisions must be carefully made: the choice of a convenient figure of merit, now discussed; and the selection of a suitable optimization algorithm, discussed in the next subsection.

The figure of merit is the output parameter in the objective function that the optimization method attempts to minimize, or maximize. Single objective optimization is claimed by the present problem, therefore a single output parameter is minimized, or maximized.

The most straightforward figure of merit is the difference, or residual, between experimental and simulated flux images. More precisely, the residual is the sum of the differences of flux in each pixel or point of computation. However, this cost function has the disadvantage of depending on the size of the spot, which varies along the day. Consequently, the use of the residual was discarded.

To overcome the problem with the residual, two alternative figures of merit were subsequently considered: the root mean square error and the cross-correlation coefficient. Both of them are independent of beam size and image resolution. The cross-correlation coefficient is a parameter frequently used in pattern recognition, e.g. Theodoridis and Koutroumbas (2009), and has been finally adopted in this study.

The cross-correlation coefficient $(C C C)$ provides a measure of the fitting between experimental and simulated flux distributions. A CCC value equal to 1 represents a perfect matching between both distributions. The optimization objective is therefore to maximize the CCC or, similarly, minimize its negative. 

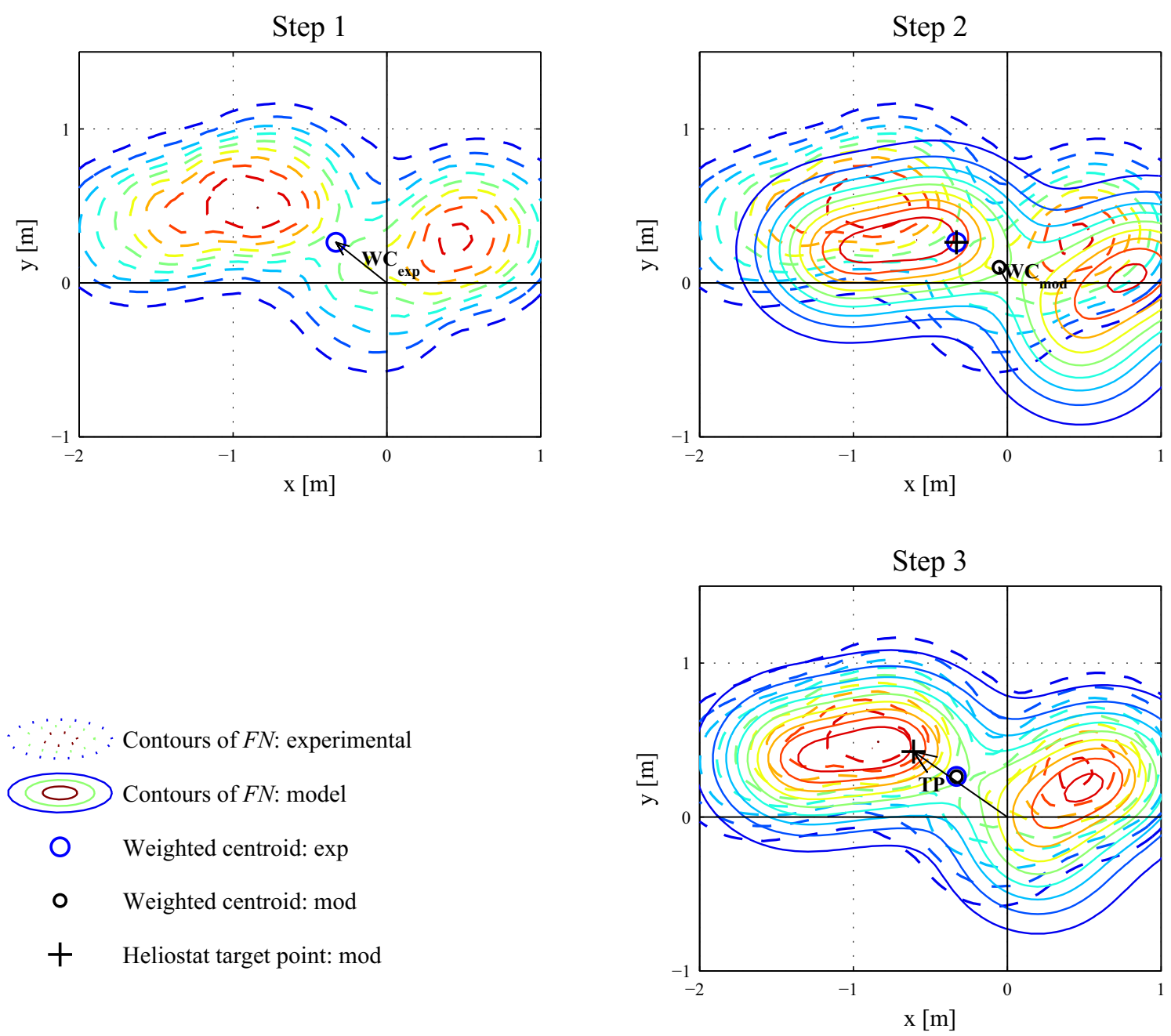

Fig. 5. Inference of heliostat target point.

The cross-correlation coefficient, also known as Pearson correlation coefficient, is calculated with Eq. (4); where: FN is the 3D matrix of normalized flux densities on the target for all the time instants (third dimension); $N_{\text {elts }}$ is the number of elements in the $3 \mathrm{D}$ matrix, which is equal to the number of pixels times the number of images used (28 in the present case); $S D$ is the standard deviation of the selected variable; and, subscripts exp and mod stand for experimental and model.

$C C C=\frac{1}{N_{\text {elts }}-1} \sum_{n=1}^{N_{\text {elts }}}\left(\frac{F N_{\text {exp }, n}-\overline{F N_{\text {exp }}}}{S D\left(F N_{\exp }\right)}\right)\left(\frac{F N_{\text {mod }, n}-\overline{F N_{\text {mod }}}}{S D\left(F N_{\text {mod }}\right)}\right)$

\subsection{Optimization algorithm}

The selection of a suitable algorithm is critical to the success of the optimization problem, in order to find a reliable solution. The three major requirements that the optimization algorithm must meet are:

1. Global solution search, avoiding local minima findings;

2. Derivative-free; and

3. Non-linearity.

The two last requirements are dictated by the nature of the objective function.
Among the plethora of global optimization methods (Arora, 2012), the solution failed under metaheuristic algorithms. Particle swarm optimization and genetic algorithm were utilized but the solution resulted very sensitive to parameters tuning. Other stochastic methods, not nature inspired, were tested. Controlled random search (CRS2) with local mutation (Kaelo and Ali, 2006) performed fairly well, even though a high population size - more than 200 samples - is needed to reach a regular solution.

Deterministic global methods have the advantage of always providing the same solution and thus avoiding multiple runs, in contrast to stochastic algorithms. At first, the Nelder-Mead simplex algorithm (Nelder and Mead, 1965) was used, leading to apparently feasible solutions in short computation time. However, simplex solution was dependent on the selected starting point, so that the first algorithm requirement was not met. Alternatively, simplex algorithm with random multistarts was tested, even though this is not a deterministic method and CRS2 with local mutation is more efficient.

The DIviding RECTangles (DIRECT) algorithm divides the search space into smaller hyper-cubes to find the global minimum (Jones et al., 1993). The dimensions with the lowest objective function values are sequentially divided into thirds. DIRECT does not require parameter tuning and convergence speed is fast. In this study the Matlab ${ }^{\circledR}$ implementation by Finkel (2003) has been utilized.

Because of its nature, DIRECT algorithm demands the definition of the bounding box within which the solution falls. Bounds in the 
Heliostat: A06

Ideal alignment (mod)

$\delta_{x, y}=0 ; \quad \sigma_{s l p}=1 \mathrm{~m} \mathrm{rad}$

$\mathrm{CCC}=\mathbf{0 . 7 2 7 6}$

$\begin{array}{cl}\therefore & \text { Experimental } \\ 0 & \text { Centroid (exp) } \\ 0 & \text { Centroid (mod) } \\ + & \text { Hel. target point (mod) }\end{array}$
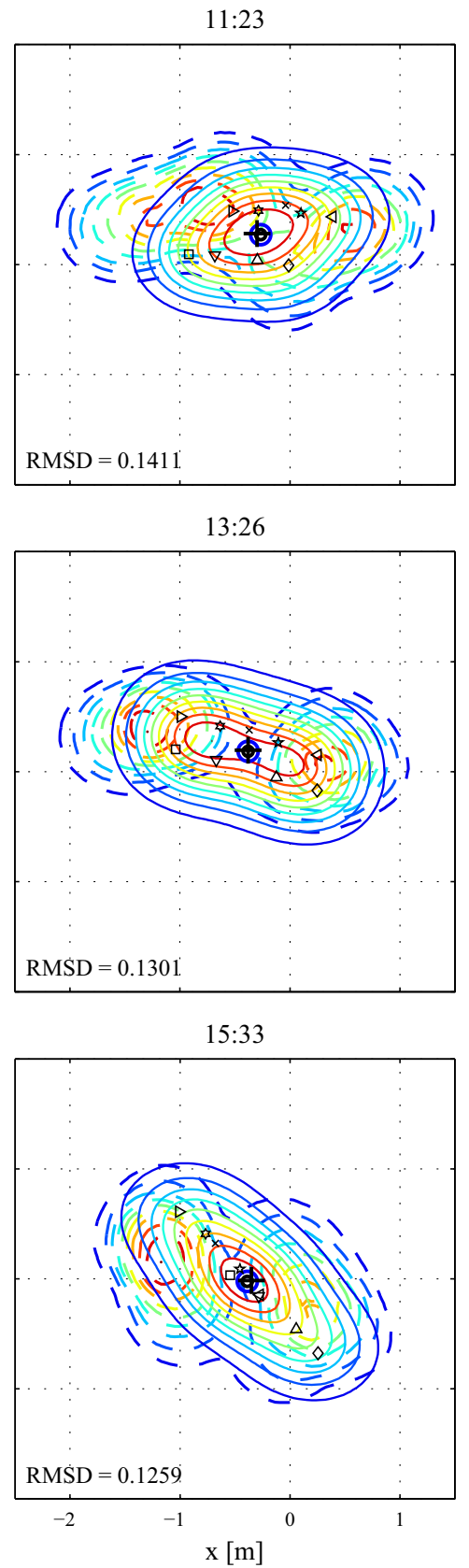

9:54
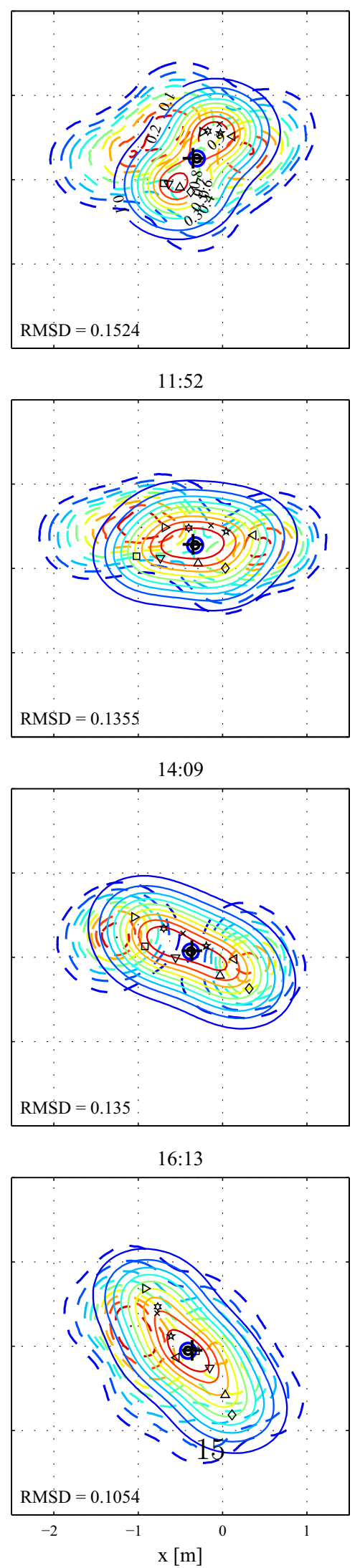

10:40
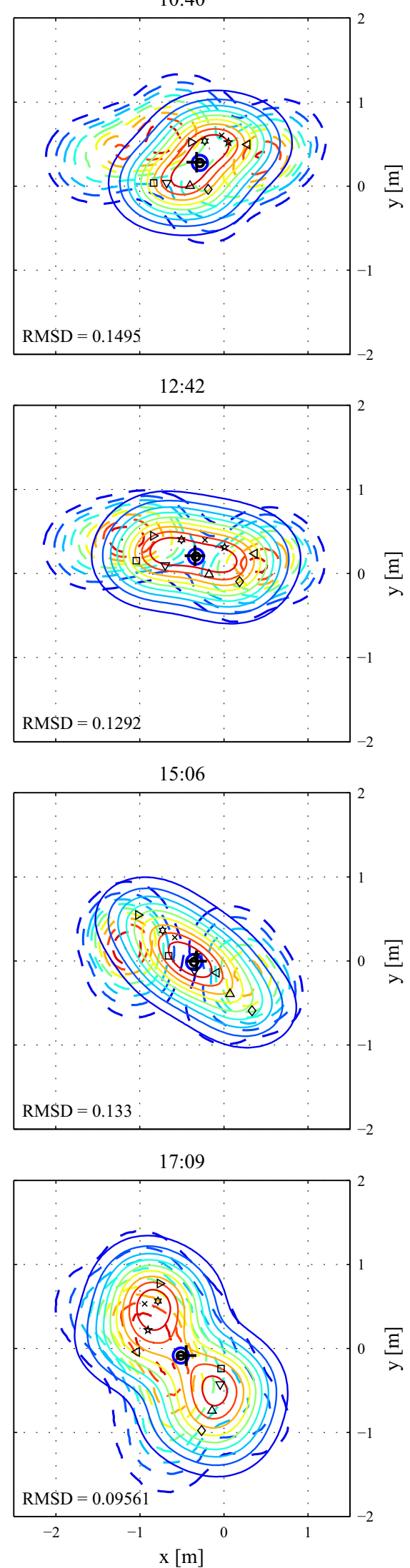

Fig. 6. Contours of normalized flux density from heliostat A06 at different CEST times in July 18, 2016. Experimental vs. model with ideal alignment. 
Table 1

Calculated canting deviations in heliostat A06.

\begin{tabular}{|c|c|c|c|c|c|c|c|c|c|}
\hline & \multicolumn{9}{|c|}{ Module } \\
\hline & 1 & 2 & 3 & 4 & 5 & 6 & 7 & 8 & 9 \\
\hline$\delta_{x}[\mathrm{mrad}]$ & -0.64 & 0.64 & 0.20 & -0.20 & 0.10 & -0.10 & 0.84 & 0.64 & 1.23 \\
\hline$\delta_{y}[\mathrm{mrad}]$ & -2.02 & -4.64 & 1.83 & 1.04 & -3.36 & -4.64 & 0.44 & -1.14 & - \\
\hline$\sigma_{s l p}[\mathrm{mrad}]$ & & & & & 1.00 & & & & \\
\hline
\end{tabular}

Heliostat: A06 Algorithm: DIRECT

\section{$\mathrm{CCC}=\mathbf{0 . 9 5 7 5}$}

\begin{tabular}{ll}
$\therefore$ & Experimental \\
\hline 0 & Model \\
$\circ$ & Centroid (exp) \\
+ & Hel. target point (mod)
\end{tabular}

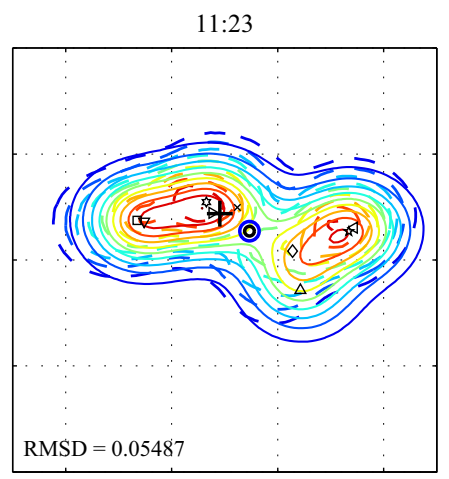

$13: 26$

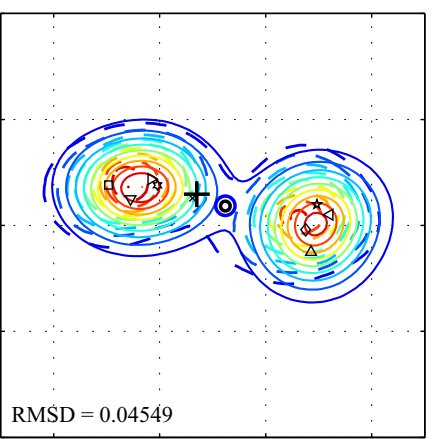

$15: 33$

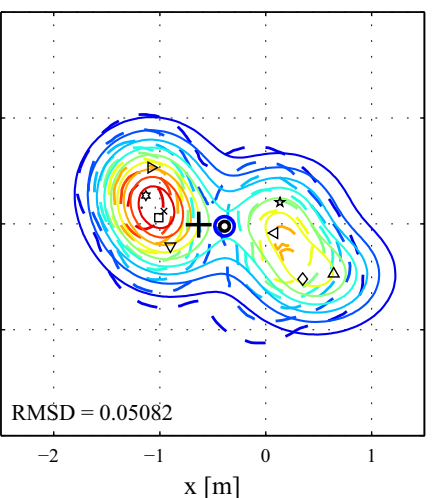

$9: 54$

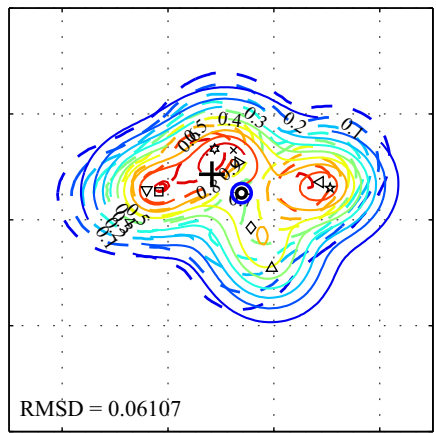

$11: 52$

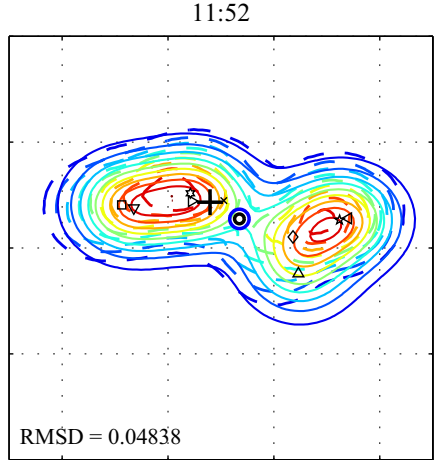

14:09

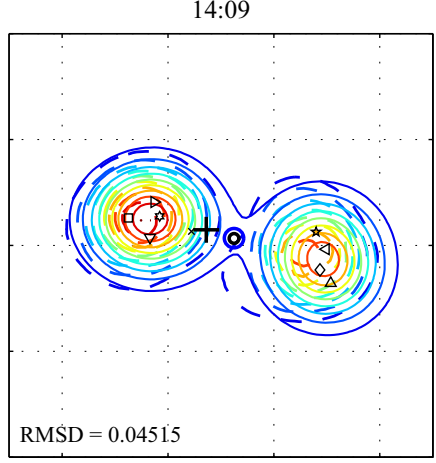

$16: 13$

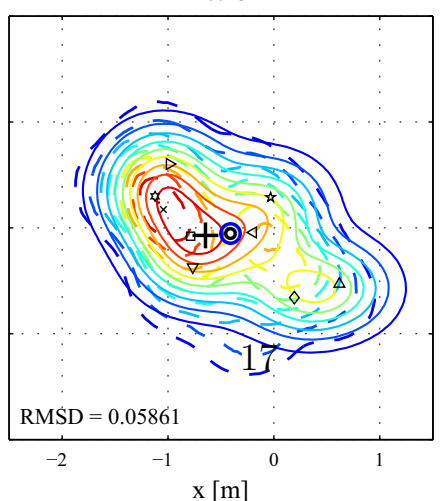

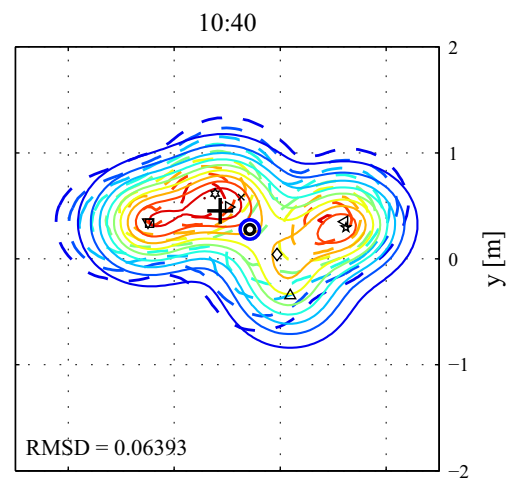

$\frac{\Xi}{\lambda}$

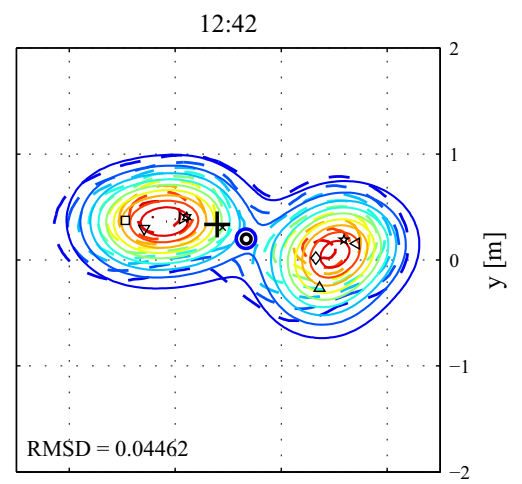

$\Xi$
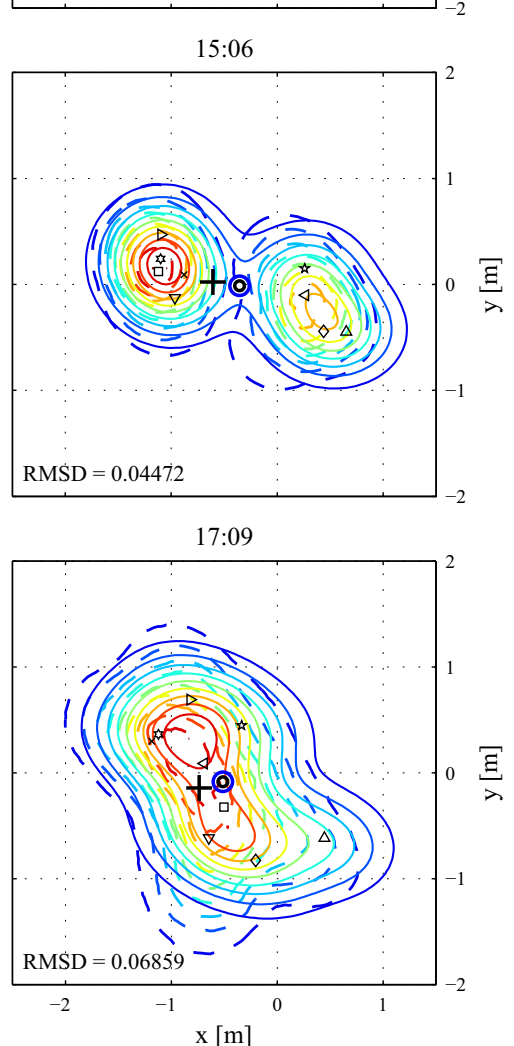

Fig. 7. Contours of normalized flux density from heliostat A06 at different CEST times in July 18, 2016. Experimental vs. model with optimized canting errors. 
search domain can be defined from the analysis of the experimental images. In heliostats where two flux spots emerge, the shift in the target points of the modules is expected to be lower than half of the distance between the two spots. As a result, in heliostat A06 a maximum angular deviation of \pm 6 mrad has been set as the bounds for all the modules in both directions, $\delta_{x}$ and $\delta_{y}$. Regarding the slope error, 0.5 and $1.5 \mathrm{mrad}$ have been established as the lower and upper bounds.

\section{Canting errors}

Before implementing the optimization procedure, experimental images were confronted against ideal ones from the optical model. Flux distributions from aligned heliostat A06 were computed, considering that no canting deviations take place in any module, and assuming slope error equal to $1 \mathrm{mrad}$. From the inspection of these images, a preliminary assessment on the canting deviations was made.

Fig. 6 represents the distributions of normalized flux density, $F N$, on the white target at 11 of the 28 instants of time considered. At each instant, it can be compared the isolines of $F N$ both measured, i.e. experimental (dashed lines), and simulated, i.e. model (solid lines). The nine $F N$ contour levels, equally spaced between 0.1 and 0.9 , are the same in both cases, as designated with the same color.

In the experimental flux distributions, two spots emerge regardless of the time instant. Even at central hours, two separate beams can be distinguished. In contrast, the flux distribution expected from adjusted heliostat A06 is grouped in a single spot. Only for instants of time near sunrise and sunshine, two peaks come up because of the increasing angle of incidence on the heliostat.

From the comparison, a clear canting problem was coming out in heliostat A06. In fact, cross-correlation coefficient was as low as 0.73 . For this reason, this heliostat was selected to test the proposed methodology.

The optimization procedure described in previous Section 3 has been applied to heliostat A06. All the 28 experimental images have been considered in the optimization in order to improve the optimization of a set of 18 unknowns. The corresponding 28 instants of time have been simulated with the optical model, now taking part of the objective function that is continuously called by the optimization algorithm.

Using DIRECT algorithm a convergent solution is reached after about 50 iterations, which represents around 10,000 evaluations of the objective function. For the selected image resolution, each run of the cost function takes on average $0.67 \mathrm{~s}$ in an Intel ${ }^{\circledR}$ Core $^{\mathrm{TM}}$ i7-870 microprocessor at $2.93 \mathrm{GHz}$. Then, less than $2 \mathrm{~h}$ of computation time are enough to reach a convergent solution on the canting deviations in the modules of a CETHEL heliostat.

Table 1 summarizes the canting errors - along with the slope error - found for heliostat A06 using the proposed methodology. Canting deviation in each module is expressed with the two axes of orientation, $\delta_{x}$ and $\delta_{y}$; except for central module 9 where adjustment around $X$ is solely allowed. Sign convention is represented in former Fig. 3. It is worth noting that almost identical results were obtained by using 14 flux images spaced along the day, instead of all the 28 images.

Fig. 7 represents the distributions of normalized flux density both measured (exp) and simulated with the canting errors found (mod) for heliostat A06. The two-spot pattern is noticeably reproduced by the optimization method. The cross-correlation coefficient CCC increases up to $95.8 \%$.

Ideally, the CCC should reach 1 to consider a perfect solution, however some factors prevent it. The optical model, grounded in
UNIZAR (Collado et al., 1986), considers an ideal spherical surface for each mirror, i.e. module. As pointed out in subSection 2.1, CETHEL module consists of three strips of parabolic mirror. The flux distribution by a CETHEL module is very similar to that of an spherical mirror with the same dimensions, even though it was proved by MCRT simulation that both distributions are not exactly the same. On the other hand, some defocus takes part on the target distribution, leading to astigmatic aberrations which are not reproduced by the optical model. In any case, correlation between experiment and model is reasonably high and the optimization algorithm provides a good estimation of the region of attraction to the perfect solution.

From the inspection of the values in Table 1, it is observed that the major deviations are around the $Y$ axes, i.e. $\delta_{y}$. In addition to this, modules in the east wing are deviated to the east, while modules in the west wing are deviated to the west, except for module 8 ; as can be figured out from sign inspection in the Table. This result is in agreement with the two lateral beams found in the central hours of the day.

In the flux maps Figures, it is indicated the root mean square deviation, RMSD, between experimental and simulated maps. In addition to this, the weighted centroids for both experimental and simulated distributions are marked with blue and black small circles, respectively. Following the instructions in subSection 3.2, both points coincide in order to infer the position of the target point, also represented in the Figures with black + marker. Similarly, the target points of every module are subtly marked in the representations; symbols used for every module are those depicted in former Fig. $1 \mathrm{~b}$.

For the sake of consistency and brevity, results for heliostat A06 have been only included in the present manuscript. It is worth mentioning that the proposed methodology has been also successfully applied to well-canted heliostats.

On the other hand, the automated code is also capable of handling any heliostat geometry with any number of rectangular facets. Even though solution uncertainty increases with the number of unknowns, the method would lead to a feasible solution, as long as care is taken to, among other things, the optimization bounds and a proper selection of the series of heliostat images.

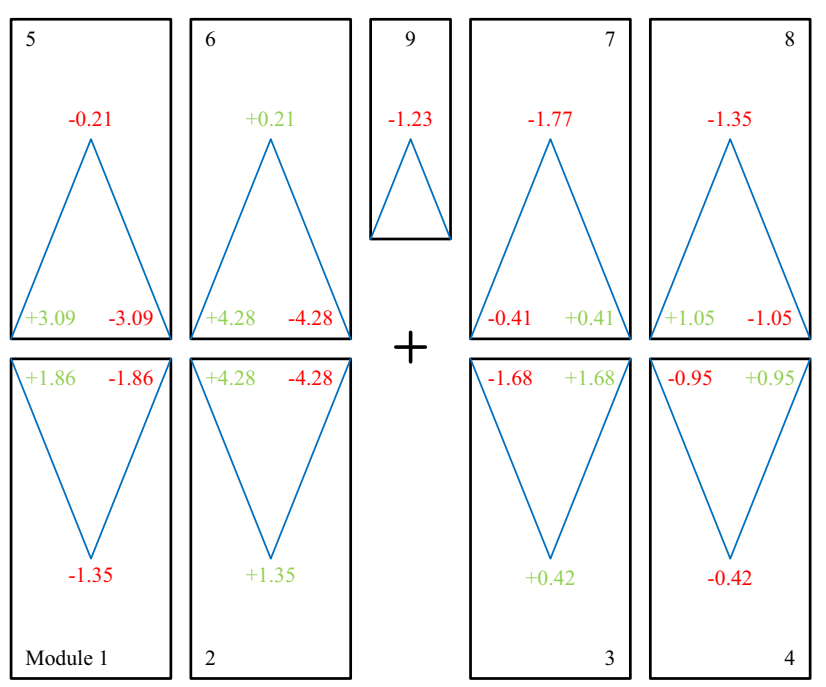

Sign convention: + forward (towards the tower); - backward (towards the ground)

Fig. 8. Adjustment of nuts in heliostat A06. Linear movement in mm. 
Before adjustment

October 21, 2016
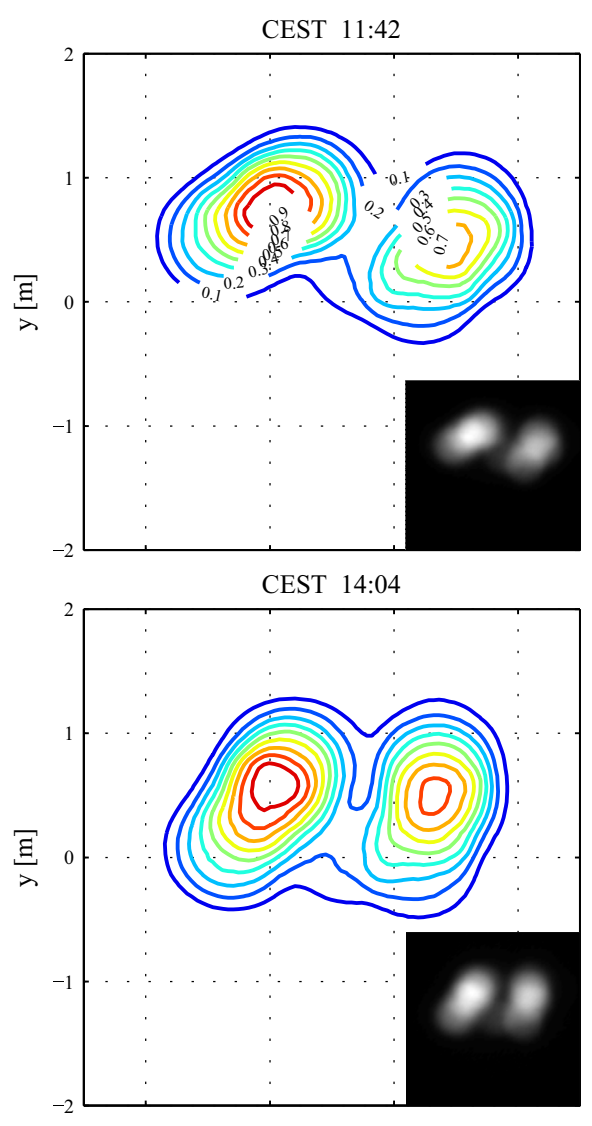

CEST 15:16

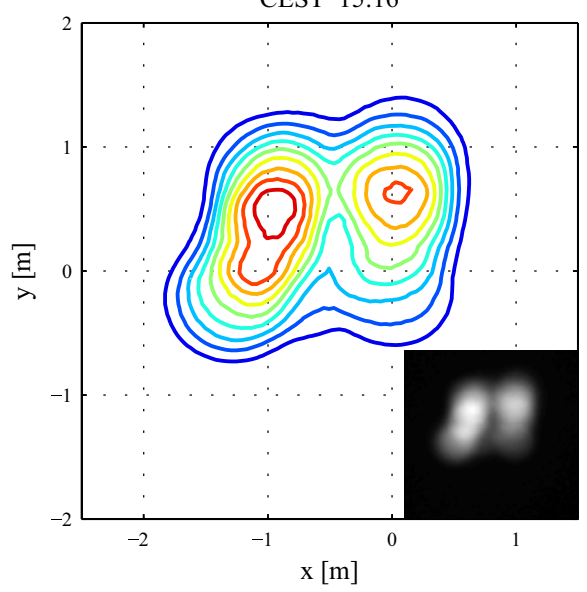

0 Experimental

$\therefore \because:$ Model

(a) Before recanting.
After adjustment

October 28, 2016
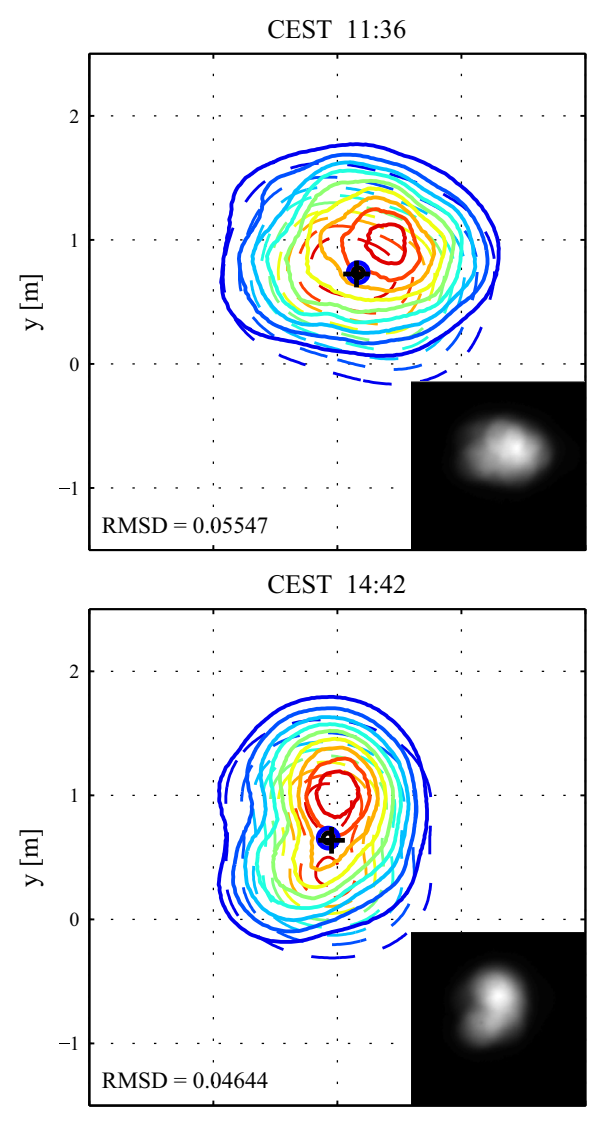

CEST 15:12

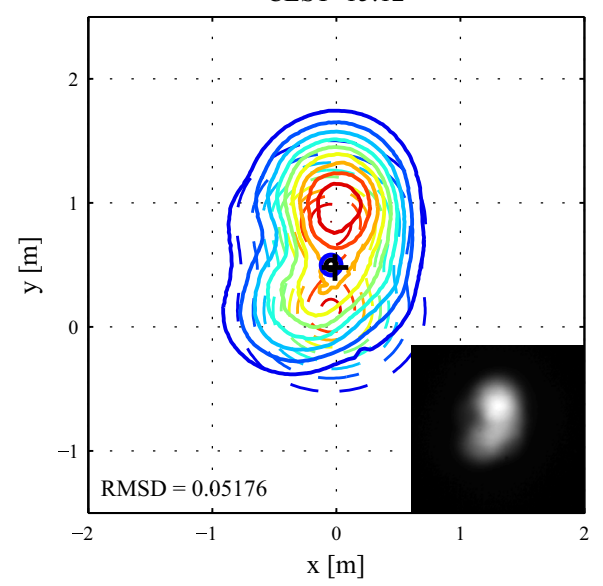

$\delta_{x, y}=0 ; \quad \sigma_{s \mid p}=1 \mathrm{mrad}$

Model: ideal alignment $\mathrm{CCC}=0.9327$

(b) After recanting.

Fig. 9. Contours of normalized flux density from heliostat A06 before and after readjustment. Grayscale captured images are shown in the lower right-hand corners.

\section{Experimental validation}

The validity of the proposed method to determine canting errors was assessed by in situ readjusting the mirror modules.

On the basis of the results presented in previous section, the modules of heliostat A06 were canted in the field. This process was performed by turning the nuts that fasten the module frame to a fixed position. From the geometry of the module, angular errors in $X$ and $Y$ directions (Table 1 ) were transformed into linear movement of the nuts.

Fig. 8 displays the displacement of the nuts prescribed for heliostat A06, with indication of the sign convention in accordance with that in Fig. 3. Some of the movements are smaller than $1 \mathrm{~mm}$, especially those in the central screws that modify $\delta_{x}$. Such small 
resolution could result in execution inaccuracies, whose experimental error has been estimated around $0.2 \mathrm{~mm}$.

Adjustment of heliostat A06 was performed on October, 2016. To assess the validity of the methodology, images on the white target were captured before - October 21 - and after - October 28 heliostat readjustment. Fig. 9 shows the experimental contours of normalized flux density before (a) and after (b) adjustment at three similar instants of time.

Three months after the initial experimental campaign and without adjusting heliostat A06, the measured flux distributions were, as expected, still spread in two spots evolving along the day, Fig. 9a. Once the heliostat was recanted, the optical quality of the heliostat significantly increased, leading to a single spot - i.e. more concentrated - on the white target, Fig. 9b.

Besides the noticeable improvement in the beam quality of heliostat A06, experimental flux maps are compared to those simulated by the optical model for the case of ideal heliostat alignment. Fig. 9b represents the contours of normalized flux density measured (solid lines) and simulated (dashed lines). Crosscorrelation coefficient reaches a large value of 93.3\%. Slight shift of the peak position respect to model prediction is found in the three instants of time. Sources of such deviation could be attributed to limitations of: the optical model that does not handle quasi-spherical mirror surface and astigmatism because of defocus; and, to a lesser extent, the optimization algorithm, and the imprecision in canting execution.

\section{Conclusions}

In this paper, a methodology to determine canting errors in real heliostats has been established. On the basis of experimental flux distributions, an optimization procedure has been defined to fit simulated distributions. Angular deviations of mirror modules have been included in the optical model, on which an objective function has been built up. The cross-correlation coefficient has been used as the figure of merit in the cost function to maximize. Because of its deterministic approach and reliability, DIRECT algorithm has been adopted to solve the optimization problem.

Such methodology has been applied to THEMIS heliostat A06, presenting poor optical quality. Along a whole day, 28 images were captured on a white target. In the absence of heliostat orientation measurements, a procedure has been developed to infer the position of the target point, founded on matching the centroids of experimental and simulated maps. The optimization problem consisting in 18 unknowns ( 17 for the angular deviations and an extra one for the mirror slope error), has been solved using DIRECT algorithm in less than $2 \mathrm{~h}$ of computation. Substantial fitting between measured flux distribution and the solution has been achieved with cross-correlation coefficients up to $95.8 \%$.

Heliostat A06, with persistent two-spot flux pattern, has been in situ readjusted according to the results obtained. The optical quality of the heliostat has been remarkably improved, leading to a much more concentrated distribution with a single spot. Compared to the result expected by the optical model, CCC rose from $72.8 \%$ to $93.3 \%$. As a consequence, the proposed methodology to find out canting errors has been validated for application to other heliostats.

\section{Acknowledgments}

The authors thank the help provided by Antoine Pérez, Yann Volut and William Baltus during the experimental phase at THEMIS solar power plant. A. Sánchez-González is also indebted to Universidad Carlos III de Madrid for the mobility grant that funded the stay at PROMES-CNRS during spring-summer 2016.

\section{References}

Arora, J.S., 2012. Global optimization concepts and methods. In: Introduction to Optimum Design. Elsevier, pp. 681-712 (Chapter 18).

Biggs, F., Vittitoe, C.N., 1979. Helios model for the optical behavior of reflecting solar concentrators. Tech. rep., Sandia National Laboratories, SAND-76-0347.

Chong, K., 2014. Non-imaging focusing heliostat. In: Tiwari, A., Valyukh, S. (Eds.) Advanced Energy Materials. John Wiley \& Sons, Inc., pp. 1-67 (Chapter 1).

Collado, F.J., Gómez, A., Turégano, J., 1986. An analytic function for the flux density due to sunlight reflected from a heliostat. Sol. Energy 37 (3), 215-234.

Finkel, D.E., 2003. DIRECT Optimization Algorithm User Guide. <http://www4.ncsu. edu/ctk/Finkel_Direct/> (accessed November 2016)

Jones, D.R., Perttunen, C.D., Stuckman, B.E., 1993. Lipschitzian optimization without the Lipschitz constant. J. Optim. Theory Appl. 79 (1), 157-181.

Jones, S.A., 1996a. A comparison of on-axis and off-axis heliostat alignment strategies. In: American Solar Energy Society Conference. Sandia National Laboratories, SAND-96-0566C, Asheville, NC (United States), pp. 95-100.

Jones, S.A., 1996b. Annual performance prediction for off-axis aligned Lugo heliostats at solar two. In: American Society of Mechanical Engineers International Solar Energy Conference. Sandia National Laboratories, SAND96-0212C, San Antonio, TX (United States).

Jones, S.A., Edgar, R.M., Houser, R.M., 1994. Recent results on the optical performance of solar two heliostats. In: American Society of Mechanical Engineers International Solar Energy Conference. Sandia National Laboratories, SAND-94-2776C, Lahaina, HI (United States)

Kaelo, P., Ali, M.M., 2006. Some variants of the controlled random search algorithm for global optimization. J. Optim. Theory Appl. 130 (2), 253-264.

Mutuberria, A., Monreal, A., Blanco, M., Sanchez, M., Ferriere, A., 2012. Modeling and structure deformation analysis of a heliostat at Mini-Pegase CNRS-PROMES facility. In: SolarPACES 2012, Marrakech (Morocco).

Nelder, J.A., Mead, R., 1965. A simplex method for function minimization. Comput. J. 7 (4), 308-313.

Ren, L., Wei, X., Lu, Z., Yu, W., Xu, W., Shen, Z., 2014. A review of available methods for the alignment of mirror facets of solar concentrator in solar thermal power system. Renew. Sustain. Energy Rev. 32, 76-83.

Sánchez-González, A., Santana, D., 2015. Solar flux distribution on central receivers: a projection method from analytic function. Renew. Energy 74 (Feb), 576-587.

Schmitz, M., Schwarzbözl, P., Buck, R., Pitz-Paal, R., 2006. Assessment of the potential improvement due to multiple apertures in central receiver systems with secondary concentrators. Sol. Energy 80 (1), 111-120.

SNL, 2013. TOPHAT. Theoretical Overlay Photographic Heliostat Alignment Technique. <https://ip.sandia.gov/technology.do/techID=16> (accessed November 2016).

Sproul, E., Chavez, K., Yellowhair, J., 2011. The development of the heliostat focusing and canting enhancement technique: an optical heliostat alignment tool for the national solar thermal test facility. In: ASME 2011 5th International Conference on Energy Sustainability, Parts A, B, and C. ASME, pp. 611-619.

Theodoridis, S., Koutroumbas, K., 2009. Template matching. In: Pattern Recognition. Academic Press - Elsevier, pp. 481-519 (Chapter 8).

USNO, 2016. Solar position. Online calculator. <http://aa.usno.navy.mil/data/docs/ AltAz.php> (accessed November 2016).

Yellowhair, J., Ho, C.K., 2010. Heliostat canting and focusing methods: an overview and comparison. ASME 2010 4th International Conference on Energy Sustainability, vol. 2. ASME, pp. 609-615.

Yuan, J.K., Christian, J.M., Ho, C.K., 2014. Compensation of gravity induced heliostat deflections for improved optical performance. J. Sol. Energy Eng. 137 (2), 021016. 